\title{
EDITORIAL
}

\section{COLONIZING THERAPEUTIC SPACE: THE OVERLOOKED SCIENCE OF DRUG HUSBANDRY}

\author{
An ever-increasing emphasis on the power of applying new technology to the early stages of \\ drug discovery might be obscuring the role that clinical science has to play in increasing the \\ number of available treatments.
}

Since, like all of us, the regulatory authorities have a last minute rush to finish off a few extra projects by the end of the year, it is only in mid-January that the true picture for worldwide drug approvals for the previous year emerges. Although the numbers are still strikingly low when compared with the number of companies actively trying to develop pharmaceuticals, the scene does look rosier than last year: the FDA approved a total of 21 New Molecular Entities for use in 2003, and the EMEA 14. Furthermore, unlike in the past few years, a substantial number of these new therapeutics act against novel targets, as discussed in the article on page 103 of this issue. This good news will undoubtedly be used throughout the coming year to suggest that we're finally beginning to see the fruits of all that investment in new processes and technology, and that this marks the beginning of an upsurge in approval rates. Hopefully so, but alongside our interest in looking to the future, are we concentrating sufficient attention on making the best use of the resources we already have?

Drug discovery and development is increasingly being depicted, at least to the public, as dependent on new technologies driving the 'discovery engine'. The rhetoric states that new screening and validation methods are about to 'revolutionize' drug discovery, delivering a bonanza of new targets and their related lead compounds, which will flow down the pipeline to become the targeted therapeutics of tomorrow. However, some worry that this emphasis on the beguiling vision of gleaming machines spitting out magic bullets serves to obscure a more pedestrian, but equally important, side of developing drugs: the science of clinical investigation. Although it seems that many drugs, far from being targeted to one specific disease, do in fact have multiple potential therapeutic applications, the scientific emphasis is currently weighted heavily in favour of the 'one drug, one disease' model. The need for a scientific approach to cultivating new uses for existing drugs, or those about to enter the market, is not given much prominence.

There is, of course, ample evidence of the value to patients of applying the techniques of clinical observation to finding new uses for drugs. Sildenafil citrate (Viagra) and thalidomide (Thalomid) are classic examples of drugs that ended up being used in entirely unexpected ways, and the expanding sphere of clinical influence for many new drugs on the market - including the highly target-specific kinase inhibitor imatinib mesylate (Glivec/Gleevec), and indeed memantine (Namenda/Ebixa), which is featured in this month's 'Fresh from the Pipeline' article on page 109 as the first licensed therapy for moderate to severe Alzheimer's disease - is testimony to the power of this approach. Using every available resource to colonize all possible 'therapeutic space' around a drug might seem an obvious thing to do, but considering the relative energies currently devoted to technological development and clinical investigation makes one doubtful that the climate supports exploring every potential avenue for existing products.

Expanding the therapeutic coverage of existing products might sound like a directive from the marketing division, whose reach back through the pipeline is already feared by scientists throughout the industry. But whereas marketing's job should properly be to help in identifying patient populations with unmet treatment needs, investing in a more experimental approach to clinical studies should be a cornerstone of the science behind drug development. To squeeze in a family analogy, my great-grandparents had one daughter every two years for 12 years (a production rate not that different from many pharma companies). When complimented once on the fine resources they had in their seven girls aged between 6 and 18 , my great-grandfather replied, "Ah yes, but one must husband one's resources." So it is with drugs, and husbandry is serious science. 